\title{
Review of: "High visit-to-visit cholesterol variability predicts heart failure and adverse cardiovascular events: a population-based cohort study"
}

nicola ferri ${ }^{1}$

1 University of Padua

Potential competing interests: The author(s) declared that no potential competing interests exist.

In the present manuscript the authors have investigated the possible association between HDL and/or LDL cholesterol variability on myocardial infarction and heart failure by analyzing an Asian population attended to a family medicine clinic in Hong Kong between 1st January 2000 to 31st December 2003 with a follow up until 31st December 2019.

The main finding is the demonstration of a link between cholesterol variability and the risk of heart failure. Major comments

1. It is not clear when the LDL and HDL cholesterol levels have been measured. The follow up is more that 15 years but when the lipid parameters have been measured? Such variability can be correct for a predetermined period of time?

2. How does the author explain the strong inverse correlation between CV LDL and cardiovascular mortality? This is opposite to new onset of heart failure and new onset of myocardial infarction. What was the cause of mortality of these patients

3. The authors conclude that "monitoring of cholesterol variability may be an attractive and viable option for risk stratification in primary care, facilitating and optimizing primary prevention of these conditions". How often it should be determined? For how long? Months, years? 